NEIP-01-005

hep-th/0106126

May 2001

\title{
Dynamical emergence of extra dimensions and warped geometries
}

\author{
Konstadinos Sfetsos \\ Institut de Physique, Université de Neuchâtel \\ Breguet 1, CH-2000 Neuchâtel, Switzerland \\ sfetsos@mail.cern.ch
}

\begin{abstract}
We present four-dimensional gauge theories in Minkowski spacetime which effectively generate in certain energy regimes five-dimensional warped geometries whereas, in general, the fifth dimension is latticized. After discussing in detail several general aspects in such theories we present a number of exactly solvable examples. We also point out how a particular case, defined in an $N$-sided polygon and having a $Z_{N}$ symmetry, has a similar realization in an appropriate supersymmetric setting with D3-branes.
\end{abstract}




\section{Introduction}

A quite attractive idea that has received a lot of attention is that our spacetime is higher than four-dimensional. This idea resonates well with string theory considerations, as well as with the more recent realization, in connection with a geometrical reformulation of the hierarchy problem, that such extra dimensions can be even macroscopic in size since this is allowed by the present day experiment evidence [1]. In most of the literature extra dimensions is an assumption that one makes. Their number, size and other properties are constrained from theoretical consistency and experimental results. In such approaches the important point is that, certain phenomena in our four-dimensional world are just "projections" of Physics in higher dimensions, where the fundamental theories are defined.

From a philosophical point of view it is quite different to demand that extra dimensions emerge dynamically and that the resulting theories are only effective descriptions of fundamental four-dimensional theories in appropriate energy regimes. Generically, such extra dimensions should emerge between certain energy scales, but the corresponding theories should look four-dimensional at very high energies in the ultraviolet (UV) and very low energies in the infrared (IR). That guarantees the four-dimensional nature of interactions at a macroscopic as well as at a microscopic level, provided the four-dimensional theories are well behaved, i.e. are renormalizable, asymptotically free etc.

In studies of gauge theories at strong coupling using the AdS/CFT correspondence, we have constructed a model which shares the desired features we just mentioned [2]. In particular, we constructed a D3-brane solution describing part of the Coulomb branch of $\mathcal{N}=4$ SYM theory with gauge group $S U(N k)$, in which vacuum expectation values (vev's) to two of the six scalars transforming in the adjoint of the gauge group were given. The scalar vev's were taken to lie in an $N$-sided polygon and in this way the original $S O(6)$ global invariance of the theory breaks to an $S O(4) \times Z_{N}$. This theory turns out to look four-dimensional for very large and very low energies and effectively five-dimensional for a wide range of energies in between. This conclusion was also verified by examining the potential energy for a quark-antiquark pair in [3]. This potential, as we will also see in this paper, has the usual Coulombic bahaviour $1 / r$ for very large and very low distances, but it behaves as $1 / r^{2}$ in between, hence confirming the effective emergence of a fifth spatial dimension.

More recently a similar idea was proposed in [4] and in [5] directly in a conventional field theory which need not be supersymmetric. A four-dimensional field theory was defined in an $N$-sided polygon with gauge group $S U(N) \times S U(k)$. Fermions provide the necessary link and transform bilinearly under nearest neighbor pairs of gauge transformations. If the dynamical energy scale for the $S U(N)$ gauge theory $\Lambda_{N}$ is much larger than that for the $S U(k)$ theory $\Lambda_{k}$, then for energies comparable to $\Lambda_{N}$ the fermions condense in pairs since at these scales each of the $S U(N)$ gauge groups becomes strong, whereas the gauge groups $S U(k)$ can still be treated perturbatively. The authors of [4] and [5] argued that the appropriate theoretical description is in terms of a latticized 
gauge theory for $S U(k)$ interacting with a $\sigma$-model that provides the link between the different sites with nearest neighbor type of interaction. The gauge group is broken down to the diagonal subgroup $S U(k)$ with the familiar Higgs mechanism. The vev's $v$ were chosen to be the same at each site and are inversely proportional to the lattice spacing. It was subsequently shown that the theory looks four-dimensional for distances smaller than the lattice spacing $1 / v$ and for distances larger than the size of the polygon $\sim N / v$. However, in between, for $1 / v \ll r \ll N / v$ the theory looks five-dimensional with the fifth dimension builded up by the Kaluza-Klein states of the large circle of radius $N / v$. The situation is the same as in [2] in the sense that in the latter case the $\sigma$-model is provided by the six scalars in the $\mathcal{N}=4 \mathrm{SYM}$ theory that transform in the adjoint of the gauge group. Also in both cases the scalars assume vev's precisely the same way. This will be shown explicitly in section 4.

We note that a similar idea of a latticized array of gauge theories has been proposed in the past in an attempt to explain the hierarchy of strengths between the electroweak and strong interactions [6]. Also, there is a close relation to lattice gauge theories with anisotropic couplings in five or higher dimensions, which, for certain values of the couplings, exhibit four-dimensional layer-type phases as it was proposed in [7] and further elaborated on, in [8, 9]. We also note recent related work that has appeared, with emphasis into more phenomenological applications in [10]-14 and some connection with non-commutative geometry in [15, 16].

The purpose of this paper is to first provide in section 2, a systematic discussion of the general case where the latticized array is not a polygon and moreover the vev's depend on the site location (the case of varying gauge coupling in treated in the appendix). This will give rise, in certain energy regimes, to a five-dimensional description in a curved warped geometry instead of just the usual Kaluza-Klein case, where the fifth dimensional is flat. In section 3 we work out some examples completely in the lattice formulation, whereas for some others we resort to the continuum approximation. We believe that these results can be further used towards constructions that are more phenomenologically oriented. Finally, in section 4, we review and refine certain aspects of the model introduced in [2] in connection with the more recent developments. We also suggest a natural mechanism for the resolution of singularities in solutions that appear in studies of the Coulomb branch of the $\mathcal{N}=4 \mathrm{SYM}$ theory. We have also written an appendix where we treat the case of varying gauge coupling according to the lattice position (but constant vev). We show that this is related to the case of varying vev's and constant gauge coupling of section 2 , by a generalization of the formulation of the usual supersymmetric quantum mechanics, but now on the discrete lattice. 


\section{The general formalism}

As in [4, 5] we start with an effective four-dimensional low energy action which is the sum of YM actions for $S U(k)$ as well as for a Higgs field that provides the necessary linking

$$
S=-\frac{1}{4(N+1) g_{4}^{2}} \int d^{4} x \sum_{j=0}^{N} \operatorname{tr}\left(F_{\mu \nu}^{j}\right)^{2}+\sum_{j=0}^{N-1} \operatorname{tr}\left(\left(D_{\mu} \Phi_{j}\right)^{\dagger} D^{\mu} \Phi_{j}\right), \quad \mu=0,1,2,3 .
$$

Each gauge term is labeled by an integer $j$, whereas the Higgs field $\Phi_{j}$ links the different gauge terms. In transforms in the $\left(\mathbf{N}_{j}, \overline{\mathbf{N}}_{j+1}\right)$ representation of the gauge group, where we note that group generators corresponding to different sites commute. Accordingly the covariant derivative is defined as $D_{\mu} \Phi_{j}=\partial_{\mu} \Phi_{j}-i A_{\mu}^{j} \Phi_{j}+i \Phi_{j} A_{\mu}^{j+1}$. The gauge coupling $(N+1) g_{4}^{2}$ is associated with each factor $S U(k)$ separately. On the other hand the coupling $g_{4}^{2}$ is associated with the diagonal $S U(k)$ that is left unbroken and dictates the physics at very low energies. We assume that there is a potential for the Higgs field $\Phi_{j}$ that allows this field to develop a vev $v_{j}=v f_{j}$, where we find it convenient to separate an energy scale $v$ from a set of numbers $f_{j}$ that characterize the strength of the vev at each site $j$. This is our main difference from [4, 5], which however will have important consequences. A similar action to (1) has appeared before in section 2 of [8], where in a five-dimensional $U(1)$ gauge theory on an anisotropic lattice, the continuum limit in the four-dimensional layer was taken, but the fifth dimension was left latticized.

We digress, to note that, the case with varying gauge coupling, and constant vev's are completely related to the case of constant gauge coupling and varying vev's that we examine here. This will be shown in the appendix.

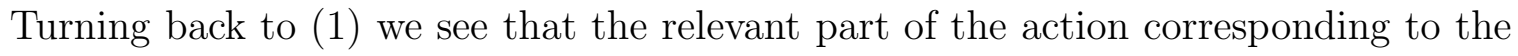
gauge field fluctuations is

$$
S=-\frac{1}{4(N+1) g_{4}^{2}} \int d^{4} x \sum_{j=0}^{N} \operatorname{tr}\left(F_{\mu \nu}^{j}\right)^{2}+v^{2} \sum_{j=0}^{N-1} f_{j}^{2} \operatorname{tr}\left(A_{\mu}^{j+1}-A_{\mu}^{j}\right)^{2} .
$$

The mass term above can be written in terms of an $(N+1) \times(N+1)$ matrix $T$ defined by writing it in the form $A_{\mu}^{t} T A^{\mu}$. Finding the mass spectrum amounts to simply diagonalizing the matrix $T$. However, this can be a cumbersome procedure except for a small number of sites. In order to develop a systematic approach we prefer to write this mass term as

$$
\sum_{j=0}^{N} A_{\mu}^{j} T_{j} A_{\mu}^{j}
$$

where the operator $T_{j}$ is defined as

$$
T_{j}=v^{2}\left(e^{-d_{j}}-1\right) f_{j}^{2}\left(e^{d_{j}}-1\right)=-4 v^{2} \sinh \left(d_{j} / 2\right) f_{j-\frac{1}{2}}^{2} \sinh \left(d_{j} / 2\right),
$$

with $d_{j}$ being the usual differential operator satisfying $\left[d_{j}, j\right]=1$. The rewriting (3) of the mass term is correct up to a term which is taken care of by the boundary conditions as discussed below. 
In principle, in order to find the spectrum of the gauge field fluctuations we need to diagonalize the operator $T_{j}$. This can be done in some cases explicitly, but of course not in general. Before we come to specific examples it is important to mention some generic properties and features. Consider the eigenvalue equation

$$
T_{j} \phi_{j}=M^{2} \phi_{j}
$$

By construction the operator $T_{j}$ is Hermitian and therefore it has real eigenvalues. In addition, since it can be written in the form $T_{j}=q_{j}^{\dagger} q_{j}$, where $q_{j}=v f_{j}\left(e^{d_{j}}-1\right)$, its spectrum is positive semi-definite, i.e. $M^{2} \geq 0$. The eigenvalue equation (5) should be supplemented with some boundary condition. In order to find what consistent boundary condition can be imposed we first realize that the equation of motion for $A_{\mu}^{N}$ as it follows with the mass term (3) is different than that following with a mass term as in (2), by the extra term $v_{N}^{2}\left(A_{\mu}^{N+1}-A_{\mu}^{N}\right)$. Translating this into a condition for the eigenvectors $\phi_{j}$ we have

$$
\text { boundary condition I : } \quad \phi_{N+1}=\phi_{N} .
$$

This boundary condition preserves the zero mode. An alternative boundary condition which does not preserve the zero mode is

$$
\text { boundary condition II : } \quad \phi_{0}=0 \text { and (or) } \quad \phi_{N}=0 .
$$

The physical motivation and consistency for such a boundary condition needs some further explanation. Let us relax the assumption that the gauge coupling is the same at all sites and introduce a "defect" by assuming that one of gauge couplings at the site $j=N$ is much smaller than the couplings at the other sites which are taken to be equal to $g_{4}^{2}$, i.e. $g_{4, N}^{2} \ll g_{4}^{2}$. Hence, in this limit, after we scale $A_{\mu}^{N} \rightarrow A_{\mu}^{N} g_{4, N}$, we see that the gauge field $A_{\mu}^{N}$ drops out of the mass term in (2). In terms of the corresponding eigenfunction $\phi_{N}$ this implies the condition $\phi_{N}=0$ in (7). A similar comment applies if we take the gauge coupling at the site $j=0$ very small, which implies then the boundary condition $\phi_{0}=0$.

Imposing the boundary condition (6) we find that there is one massless eigenvalue corresponding to the zero model, as well as $N$ massive ones. We emphasize that this procedure of finding the eigenvalues is completely equivalent to simply diagonalizing the $(N+1) \times(N+1)$ matrix $T$ defined before by writing the mass term in (2) in the form $A_{\mu}^{t} T A^{\mu}$. We can always construct a set of $N+1$ eigenvectors $\left\{\phi_{j, n}, n=0,1, \ldots, N\right\}$ of the operator $T_{j}$ that obey the eigenvalue equation (5) as well as the orthogonality and completeness relations

$$
\sum_{j=0}^{N} \phi_{j, n} \phi_{j, m}^{*}=\delta_{n, m}, \quad \sum_{n=0}^{N} \phi_{j, n} \phi_{k, n}^{*}=\delta_{j, k}
$$

The normalized wavefunction corresponding to the zero mode is $\phi_{j, 0}=(N+1)^{-1 / 2}$ with $M_{0}=0$. 
On the other hand, imposing one of the boundary condition in (7) we find that there are $N$ massive eigenvalues, but the zero mode is projected out of the spectrum. Then, in the finite sums in (8) we exclude the corresponding terms.

Lets us introduce a source term corresponding to a unit charge at the origin of the coordinate system and at the $k$-th site. Then, the differential equation that determines the potential energy at site $j$ due to a charge located at site $k$ is given by

$$
\nabla_{3}^{2} V_{j, k}-T_{j} V_{j, k}=4 \pi(N+1) g_{4}^{2} \delta_{j, k} \delta^{(3)}(\mathbf{x}) \text {. }
$$

We may expand $V_{j}$ is terms of the basis eigenvectors of the differential operator $T_{j}$ as $V_{j, k}(r)=\sum_{n} \tilde{V}_{n, k}(r) \phi_{j, n}$. Using (8) we eventually arrive at

$$
V_{j, k}(r)=-\frac{(N+1) g_{4}^{2}}{r} \sum_{n=0}^{N} \phi_{j, n} \phi_{k, n}^{*} e^{-M_{n} r} .
$$

We may discuss quite generally, several features for the behaviour of the potential in (10). For instance, at distances much larger that the typical size of the array, i.e. $r \gg N / v$, obviously only the zero mode, if it exists, contributes to the sum in (10). Hence we have that

$$
\text { Zero mode exists : } \quad V_{j, k}(r) \simeq-\frac{g_{4}^{2}}{r}, \quad \text { for } \quad r \gg N / v
$$

Therefore, at low energies we obtain the usual Coulombic behaviour with coupling constant $g_{4}^{2}$ (we ignore possible slow variations due to the running of couplings). This is the expected behaviour for a four-dimensional gauge theory for $S U(k)$ which is the unbroken diagonal subgroup of the original $N+1$ copies of $S U(k)$. This behaviour is universal for all sites due to the fact that the wavefunction for the zero mode has the same value at all sites. For the cases that the zero mode does not exist the typical behaviour is

$$
\text { Zero mode does not exist : } \quad V_{j, k}(r) \sim-\frac{g_{4}^{2} \phi_{j, 1} \phi_{k, 1}^{*}}{r} e^{-M_{1} r}, \quad \text { for } \quad r \gg N / v,
$$

where the inverse of the lowest eigenvalue, which is expected to be comparable to the typical size of the array, i.e. $M_{1}^{-1} \sim N / v$, sets the range of the Yukawa interaction. Its

\footnotetext{
${ }^{1}$ For completeness, we note that, when the eigenvectors $\phi_{j, n}$ form a complete but not orthonormal set, we may follow the procedure of Gram-Schmidt in order to construct such a set. Otherwise, (8) is replaced by

$$
\sum_{j=0}^{N} \phi_{j, n} \phi_{j, m}^{*}=A_{m n}, \quad \sum_{n, m=0}^{N} \phi_{j, n} A_{n m}^{-1} \phi_{k, m}^{*}=\delta_{j, k} .
$$

where $A_{m n}$ is some $(N+1) \times(N+1)$ Hermitian matrix. Consequently, 10) is replaced by

$$
V_{j, k}(r)=-\frac{(N+1) g_{4}^{2}}{r} \sum_{n, m=0}^{N} \phi_{j, n} A_{n m}^{-1} \phi_{k, m}^{*} e^{-M_{n} r} .
$$


effective overall strength depends on the sites.f In contrast, for distances $r \ll 1 / v$ all modes in the sum in (10) contribute equally. After using the completeness relation in (\$) we obtain

$$
V_{j, k}(r) \simeq-\frac{(N+1) g_{4}^{2}}{r} \delta_{j, k}, \quad \text { as } \quad r \ll 1 / v .
$$

This is the expected behaviour for a four-dimensional theory at high energies where we have $N+1$ independent copies of gauge theories for $S U(k)$, each with gauge coupling $(N+1) g_{4}^{2}$. Since, the various copies are independent the potential is non-vanishing only for charges charged with the same $S U(k)$ factor, i.e. $j=k$. For charges charged with respect to different $S U(k)$ factors where $j \neq k$, the potential has an expansion in powers of $r$ with leading term of order $g_{4}^{2} v N$ for site-separation of order 1 , and $g_{4}^{2} v / N$ for siteseparation of order $N$. Hence, the magnitude of the force between charges located at the same site is much larger than that between charges located at different sites. In the latter cases, higher loop diagrams that contribute may be considered as, depending on the value of the coupling constant, they may give comparable contributions (a similar comment has been made for a specific example in [4).

We conclude our general discussion with further remarks on the general behaviour of the potential as a function of $r$. First, notice that, as it follows from (13), we have implicitly chosen opposite charges for the two charged particles with respect to the diagonal $S U(k)$ subgroup that prevails at low energies. The question is whether it is possible in some models to have a repulsive instead of attractive force for some $v r \sim 1$, although of course eventually the force has to turn attractive according to (13). Certainly, the possibility of an repulsive force for some range of $r$ is excluded for charges at the same site since all terms in (10) come with the same sign when $j=k$. However, for $j \neq k$ not all terms have the same sign and the possibility of a repulsive force is not excluded for some range of the values for $r$. Nevertheless, in all of our examples in this paper the force remains attractive for all $r$, in agreement with physical expectations.

\subsection{Continuum limit and a fifth dimension}

A quite interesting behaviour occurs for intermediate distances, which are much larger that the lattice spacing, but also much smaller than its typical size $\sim N / v$, namely for $1 / v \ll r \ll N / v$. Here we assume that $N \gg 1$. Then we may approximate the lattice by a continuum with fifth space-variable $x_{5}=j / v$. Then the differential operator $T_{j}$ is well approximated by $T\left(x_{5}\right)=-\partial_{5} f\left(x_{5}\right)^{2} \partial_{5}$, where $f\left(x_{5}\right)$ is the continuum limit of the sequence of numbers $\left\{f_{j}\right\}$ which is assumed to exist. Then, the continuous limit of the action (2) is written as

$$
S=-\frac{1}{4 g_{5}^{2}} \int d^{4} x d x_{5} \operatorname{tr}\left(F_{\mu \nu}^{2}+f\left(x_{5}\right)^{2}\left(\partial_{5} A_{\mu}\right)^{2}\right), \quad g_{5}^{2}=N g_{4}^{2} / v .
$$

\footnotetext{
${ }^{2}$ In (14) the strength of the Yukawa interaction is constant. This is due to the discreteness of the spectrum. In cases where the continuum limit is considered this strength is $r$-dependent, giving rise to an overall factor for $e^{-M_{1} r}$ which is proportional to $1 / r^{3 / 2}$ instead of $1 / r$.
} 
This can be reinterpreted as the five-dimensional action $-\frac{1}{4 g_{5}^{2}} \int d^{5} x \sqrt{-G} \operatorname{tr}\left(F_{M N}^{2}\right)$, where $M=(\mu, 5)$ and in the gauge $A_{5}=0$, defined in the background with a metric given by the line element

$$
d s^{2}=f\left(x_{5}\right)^{2} \eta_{\mu \nu} d x^{\mu} d x^{\nu}+d x_{5}^{2} .
$$

Hence, what we have derived is that there is an energy regime where we may safely use a five-dimensional gauge theory defined in a curved, in general, warped spacetime, in order to compute physical processes in a gauge theory in the usual four-dimensional Minkowski spacetime. The four-dimensional gauge theory is renormalizable whereas the five-dimensional one is not and only emerges as an effective description in the appropriate energy regime.

We make the important note that the regime where $1 \ll v r \ll N$ is a strict continuum limit where the operator $T_{j}$ is well approximated by the second order differential operator $T\left(x_{5}\right)=-\partial_{5} f\left(x_{5}\right)^{2} \partial_{5}$. Higher terms that appear in the derivative expansion of $T_{j}$ are neglected. However, there is a less restrictive continuum limit that retains all such higher derivative terms. As it will also be confirmed in the examples below, this limit is attained for $v r \ll N$ and therefore is valid for distances $v r \simeq 1$, where the approximation that leads to (16) fails.

For future convenience it is better to use the variable $z$ instead of $x_{5}$, with change of variables as $d x_{5}=e^{A} d z$. Also we will rename $f^{2}$ by $e^{2 A}$. The equation determining the potential between two charges is still given by (9) with $T_{j}$ replaced by $T\left(x_{5}\right)$. The corresponding eigenvalue equation for this operator becomes

$$
T \phi_{n}=-e^{-A} \partial_{z} e^{A} \partial_{z} \phi_{n}(z)=M_{n}^{2} \phi_{n}(z),
$$

where we have used the variable $z$ instead of $x_{5}$. The orthogonality and completeness relations read

$$
\int d z e^{A} \phi_{n}(z) \phi_{m}^{*}(z)=\delta_{n, m}, \quad \sum_{n=0} \phi_{n}(z) \phi_{n}^{*}\left(z^{\prime}\right)=e^{-A} \delta\left(z-z^{\prime}\right) .
$$

As in the discrete case, we may distinguish the following two cases: If the spectrum of the operator $T$ has a mass gap then the behaviour of the potential between charges is, for large distances, of the Yukawa-type, whereas if there is no such mass-gap we have a power-law type of behaviour.

We note that, even though for notational purposes we have used the discrete set of number $\{n \in Z\}$ in order to label our states, the spectrum can be discrete of continuous depending on the function $A(z)$. In order to check that it is convenient to cast the differential equation in (18) as a Schrödinger differential equation. Indeed, after defining $\phi_{n}=e^{-A / 2} \Psi_{n}$ we obtain

$$
-\partial_{z}^{2} \Psi_{n}+V_{\text {Sch. }} \Psi_{n}=M_{n}^{2} \Psi_{n}, \quad V_{\text {Sch. }}=\frac{1}{4}\left(\partial_{z} A\right)^{2}+\frac{1}{2} \partial_{z}^{2} A .
$$

This potential has the form of the potentials appearing in supersymmetric quantum mechanics [17] with corresponding superpotential $W=-\frac{1}{2} \partial_{z} A$. Hence, the spectrum 
is positive semi-definite as already expected from the fact that it originates from the spectrum of the operator $T_{j}$ which we have already argued that it is positive semi-definite.

If the Schröndinger potential in (20) can be neglected for some wide range of values of the variable $z$, then in this range the wavefunctions are approximated by plane-waves as $\Psi_{k} \simeq 1 / \sqrt{2 \pi} e^{i k z}$. Then, using that $\phi_{k}=e^{-A / 2} \Psi_{k}=f\left(x_{5}\right)^{-1 / 2} \Psi_{k}$ we obtain that the potential between charges at positions $z$ and $z^{\prime}$ in the fifth dimension, is given by

$$
V_{z, z^{\prime}}(r) \simeq-\frac{g_{5}^{2}}{\pi} \frac{e^{-\frac{1}{2}\left(A(z)+A\left(z^{\prime}\right)\right.}}{r^{2}+\left(z-z^{\prime}\right)^{2}} .
$$

This behaviour resembles the usual Kaluza-Klein case, but with an effective coupling that depends on the fifth dimension. This is due to the fact that the effective fifth

dimension that emerges is not flat. Such behaviour will be encountered in some of the explicit examples below.

\section{Solvable examples}

The examples that we specifically work out in this section belong to two categories: Those where we work directly in the lattice formulation and those where the continuous approximation based on (16) is employed from the very beginning. We first consider the case of a periodic array on a polygon that has been considered before in this context in [4, 5] and its supersymmetric variant already within the context of the AdS/CFT correspondence in [2]. In our second example the periodicity condition is relaxed, resulting into a model that has a discrete mass spectrum, but no zero mode. Both cases share the feature that the vev's are the same at all sites. Our third example has vev's that vary with the site position, but even so it turns out that is is completely solvable with the help of Laguerre polynomials. Finally, within the continuous approximation we consider two classes of examples with varying vev's. Both have a continuous spectrum but one of them has a mass gap.

\subsection{Periodic array on a polygon}

In this example we give the same vev at all sites, i.e. $v_{j}=v$. Moreover, because of the $Z_{N+1}$ symmetry of the array, we require periodicity of the wave function as $\phi_{j+N+1}=$ $\phi_{j}$. Since we have that $f_{j}=1$ the operator in (荬) is simply $T_{j}=-4 v^{2} \sinh ^{2}\left(d_{j} / 2\right)$. This commutes with the differential operator $d_{j}$ and therefore they have the same set of eigenfunctions which when properly normalized read

$$
\phi_{j, n}=\frac{1}{\sqrt{N+1}} e^{2 \pi i \frac{j n}{N+1}}, \quad j=0,1, \ldots, N, \quad n=0,1, \ldots, N .
$$

The $N+1$ mass eigenvalues are given by

$$
M_{n}=2 v \sin \left(\frac{\pi n}{N+1}\right), \quad n=0,1, \ldots, N,
$$


where we also observe that a zero mode exists. Since $\left|\phi_{j, n}\right|=1 / \sqrt{N+1}$ the potential (10) is the same for all sites, as expected due to the $Z_{N+1}$ symmetry of the array. Even in this simple case, it does not seem possible to perform the sum in (10) explicitly. Nevertheless, the behaviour for $r \gg N / v$ and $r \ll 1 / v$ is that predicted on general grounds by (13) and (15), respectively.

The continuum limit holds for $v r \ll N$ and $j-k \ll N$, Then the original discrete periodic array is replaced by a continuum periodic array, but we retain all terms in the derivative expansion of the operator $T_{j}$. The expression for the potential turns out to be given by the following integral

$$
V_{j, k}(r)=-2 \frac{g_{4}^{2} N}{\pi r} \int_{0}^{\pi / 2} d \varphi \cos (2(j-k) \varphi) e^{-2 v r \sin \varphi} .
$$

We may evaluate this in terms of generalized hypergeometric functions, but we will refrain from presenting the result since the resulting expressions are quite complicated.

If we further restrict to distances $1 \ll v r \ll N$ we find the behaviour

$$
V_{j, k}(r) \simeq-\frac{g_{5}^{2}}{\pi} \frac{1}{r^{2}+\left(x_{5}-x_{5}^{\prime}\right)^{2}}, \quad \text { for } \quad 1 / v \ll r \ll N / v,
$$

where $x_{5}=j / v$ and $x_{5}^{\prime}=k / v$. This is the regime where the description in terms of a five-dimensional theory in flat space (since $f\left(x_{5}\right)=1$ ) becomes appropriate. Then the continuum periodic array is replaced by a an infinite continuum array. In that case the properly normalized wavefunctions are $\phi_{k}\left(x_{5}\right)=e^{i k x_{5}} / \sqrt{2 \pi}$, with $z$ taking values in the whole real line, whereas the mass spectrum $M^{2}=k^{2}$ is continuous. Then using that $V=-g_{5}^{2} / r \int_{-\infty}^{\infty} d k e^{-|k| r} /(2 \pi)$ we exactly reproduce (25). This is the typical Kaluza-Klein behaviour corresponding to the five-dimensional effective theory (16) and was already found in [4, 5].

There is in addition the scale regime $v r \gg 1$ where the original discrete periodic array is replaced by a infinite, but nevertheless discrete array. Then the potential is given by an infinite sum which can be computed exactly

$$
\begin{aligned}
V_{j, k}(r) & =-\frac{g_{4}^{2}}{r} \sum_{n=-\infty}^{\infty} e^{2 \pi i(j-k) n / N} e^{-2 \pi v r|n| / N}=-g_{5}^{2} \sum_{n=-\infty}^{\infty} \frac{1}{r^{2}+(j-k-N n)^{2} / v^{2}} \\
& =-\frac{g_{4}^{2}}{r} \frac{\sinh (2 \pi r v / N)}{\cosh (2 \pi r v / N)-\cos (2 \pi(j-k) / N)} .
\end{aligned}
$$

This expression interpolates between those in (25) for $1 \ll v r \ll N$ and (13) for $v r \gg N$. In the former case the corrections to (25) are in powers of $v r / N$, whereas in the latter case the corrections to (13) are Yukawa-like, corresponding to the Kaluza-Klein modes of a reduction to a circle of radius $N / v$.

\footnotetext{
${ }^{3}$ However, we may easily prove that for $r \ll 1 / v$, the behaviour of the effective potential is $V_{j, k} \simeq$ $-\delta_{j, k} g_{4}^{2}(N+1) / r-\pi v g_{4}^{2} /(N+1)\left(\sin ^{2}(\pi(j-k) /(N+1))-\left(\frac{1}{2} \pi /(N+1)\right)^{2}\right)^{-1}+\cdots$. This is in accordance with the general behaviour that we have stated after eq. (15).
} 


\subsection{Relaxing the periodicity condition}

As in the previous example we give the same vev to all sites, but we no longer impose the periodicity condition. Instead, we impose the boundary condition (7) that the wave function $\phi_{j}$ vanish at the endpoints, namely that $\phi_{0}=\phi_{N}=0$. The properly normalized wavefunctions that form a complete set are

$$
\phi_{j, n}=\sqrt{\frac{2}{N}} \sin (\pi j n / N), \quad j=0,1, \ldots, N, \quad n=1,2, \ldots, N-1
$$

The mass spectrum is given by

$$
M_{n}=2 v \sin \left(\frac{\pi n}{2 N}\right), \quad n=1,2, \ldots, N-1,
$$

where the zero mode is projected out. With these eigenfunctions and eigenvalues the sum in (10) cannot be computed exactly. Nevertheless, we may show that the behaviour for $r \gg N / v$ and $r \ll 1 / v$ is that predicted on general grounds by (13) and (15), respectively.

In the limit $v r \gg 1$ and for $N \gg 1$ we have that (we set $L=N / v$ )

$$
\begin{aligned}
V_{j, k}(r) & =-\frac{2 g_{4}^{2}}{r} \sum_{n=1}^{\infty} \sin (\pi j n / N) \sin (\pi k n / N) e^{-\pi v n r / N} \\
& =-\frac{g_{4}^{2}}{r} \frac{\sin \left(\frac{\pi j}{N}\right) \sin \left(\frac{\pi k}{N}\right) \sinh \left(\frac{\pi r}{L}\right)}{\left[\cosh \left(\frac{\pi v r}{N}\right)-\cos \left(\frac{\pi(j+k)}{N}\right)\right]\left[\cosh \left(\frac{\pi v r}{N}\right)-\cos \left(\frac{\pi(j-k)}{N}\right)\right]} .
\end{aligned}
$$

For $r \ll x_{5} \sim x_{5}^{\prime} \ll L$, where $x_{5}=j / v$ and $x_{5}^{\prime}=k / v$, we obtain the same behaviour as in (25). Also

$$
V_{x_{5}, x_{5}^{\prime}} \simeq-\frac{4 g_{4}^{2} L x_{5} x_{5}^{\prime}}{\pi r^{4}}, \quad x_{5}, x_{5}^{\prime} \ll r \ll L,
$$

which is a behaviour similar to that of a seven-dimensional theory. Finally, for distances much larger than the length $L$ we have

$$
V_{j, k} \simeq-\frac{2 g_{4}^{2}}{r} \sin (\pi j / N) \sin (\pi k / N) e^{-\pi r / L}, \quad \text { for } \quad r \gg L
$$

in accordance with the general formulae (13).

There is also a continuum limit for distances $r v \ll N$, that goes beyond the two derivative approximation (14)

$$
V_{j, k}(r)=-2 \frac{g_{4}^{2} N}{\pi r} \int_{0}^{\pi / 2} d \varphi \sin (2 j \varphi) \sin (2 k \varphi) e^{-2 v r \sin \varphi} .
$$

Again this is expressible in terms of generalized hypergeometric functions. 


\subsection{Array with varying vev's}

Cases with vev's varying according to the site position are in general more difficult to handle for increasing site number $N$. However, let us consider the case of $v_{j}=v \sqrt{j}$, $j \geq 1$. 1 Though not obvious, it is possible to completely determine in general the eigenvalues and eigenfunctions of the operator $T_{j}$ which now equals

$$
T_{j}=v^{2}\left(e^{-d_{j}}-1\right) j\left(e^{d_{j}}-1\right) .
$$

As in some quantum mechanical problems it is convenient to pass to the momentum representation where the operator $d_{j}$ is just a number denoted by ip and the site position $j$ becomes the differential operator $i \partial_{p}$. Then the eigenfunctions in the momentum representation satisfy the differential equation

$$
i v^{2}\left(e^{i p}-1\right) \partial_{p}\left(\left(e^{i p}-1\right) \phi_{p}\right)=M^{2} \phi_{p}
$$

with properly normalized solutionf

$$
\begin{array}{r}
\phi_{p}=\frac{i}{\sqrt{\pi}} \frac{e^{i m^{2} / 2 \cot (p / 2)}}{e^{i p}-1}=\frac{1}{2 \sqrt{\pi} i} e^{i m^{2} / 2 \cot (p / 2)}(1+i \cot (p / 2)), \\
0 \leq p \leq 2 \pi, \quad m=M / v .
\end{array}
$$

In order to compute the potential between charges we need the wavefunction in the position representation $\phi_{j}$. This is given by

$$
\phi_{j}=\sqrt{\frac{1}{\pi}} \int_{0}^{2 \pi} d p \sin (j p) \phi_{p} .
$$

After changing variables as $x=\cot (p / 2)$ we have to compute the integral in (36) which takes the form

$$
\phi_{j}=-\frac{1}{2 \pi} \int_{-\infty}^{\infty} d x\left(\frac{(x+i)^{j-1}}{(x-i)^{j+1}}-\frac{(x-i)^{j-1}}{(x+i)^{j+1}}\right) e^{i m^{2} x / 2}(1+i x) .
$$

The integrand has poles at $x= \pm i$ in the complex plane and we may compute it using standard methods in complex analysis. Since $m^{2}>0$ we may choose the contour of integration to close in the upper half plane and to include $x=i$. Then, only the first term in the integrand in (37) contributes. The result is

$$
\begin{aligned}
\phi_{j} & =\frac{1}{(j-1) !} \frac{\partial^{j-1}}{\partial x^{j-1}}\left[e^{-m^{2} x / 2}(x+1)^{j-1}\right]_{x=1} \\
& =e^{-m^{2} / 2} \sum_{n=0}^{j-1} \frac{(j-1) !\left(-m^{2}\right)^{n}}{(j-1-n) !(n !)^{2}} .
\end{aligned}
$$

\footnotetext{
${ }^{4}$ For convenience, we have shifted the range of $j$ by one. Hence, $j$ starts from $j=1$ and not from $j=0$ as in the general formulation before. We take this into account in the rest of this subsection.

${ }^{5}$ We note that the case of an array with $v_{j}=v j, j \geq 1$ is also solvable by applying the same idea. Indeed, in this case after we substitute $\phi_{p}=(1-q)^{\mu} F(q)$, where $q=\cos ^{2}(p / 2)$ and $\mu=1 / 4+$ $i / 2 \sqrt{m^{2}-1 / 4}$, we obtain that $F(q)$ satisfies a hypergeometric equation with $a=b=\mu$ and $c=1 / 2$ in the standard notation. We also note that, normalizability of $\phi_{p}$ requires that the mass spectrum has a gap, $M_{\text {gap }}=v / 2$.
} 
One recognizes the last sum as an expression for the Laguerre polynomials $L_{j-1}\left(m^{2}\right)$. Therefore

$$
\phi_{j}=e^{-m^{2} / 2} L_{j-1}\left(m^{2}\right), \quad j=1,2, \ldots .
$$

Having obtained this solution in a systematic way, it is important to verify directly that (39) indeed satisfies the eigenvalue equation $T_{j} \phi_{j}=M^{2} \phi_{j}$, where $T_{j}$ is given by (33). This is quite straightforward, using the recursion relations for the Laguerre polynomials, $L_{j-1}\left(m^{2}\right)$.

\subsubsection{The limit of infinite array}

In the case that the array is large, so that $N \gg 1$, boundary effects can be neglected for $v r \ll N$. Then the mass spectrum is continuous with no mass gap $\left(M^{2} \geq 0\right)$. Using the orthogonality and completeness properties of the Laguerre polynomials for $m^{2} \in(0, \infty)$ we see that (8) are satisfied. The potential between charges at sites $j$ and $k$ is then

$$
V_{j, k}(r)=-2 \frac{g_{4}^{2} N}{r} \int_{0}^{\infty} d m m e^{-m^{2}-m v r} L_{j-1}\left(m^{2}\right) L_{k-1}\left(m^{2}\right), \quad \text { for } \quad v r \ll N .
$$

For specific values for $j$ and $k$ we may give the result in terms of the error function $\operatorname{erf}(v r / 2)$ and powers of $v r$, but we will not present any results explicitly.

It is easy to see that for $v r \ll 1$ we obtain (15) whereas for $v r \gg 1$ and small values for $j$ and $k$ we obtain an $1 / r^{3}$ fall-off for the potential as

$$
V_{j, k}(r) \simeq-2 \frac{g_{4}^{2} N}{v^{2} r^{3}}, \quad \text { for } \quad j-k \ll v r \ll N
$$

This is characteristic of the behaviour of a six-dimensional theory.

The limit of site position $j \gg 1$ and $m^{2} \ll 1$ is also of particular interest. Then using properties of the Laguerre polynomials we find that

$$
\phi_{j} \simeq J_{0}(2 m \sqrt{j}), \quad \text { as } \quad j \gg 1, m^{2} \ll 1 \text { and } j m^{2}=\text { finite },
$$

where $J_{0}$ is the zeroth order Bessel function. This is the result that one would have obtained from the five-dimensional two-derivative effective theory based on (16), as we will shortly verify (see footnote 6 below).

\subsubsection{Finite arrays}

In the case that the array is finite with $j=1,2, \ldots, N+1$ we have to consider boundary effects. There are two choices, one projecting out the zero mode and one preserving it.

Projecting out the zero mode: We impose the boundary condition (7) that the wavefunction vanishes at the end, namely that $\phi_{N+1}=0$. This condition determines the allowed values for $m_{n}^{2}$ as the $N$ zeros of the corresponding Laguerre polynomial $L_{N}$

$$
L_{N}\left(m_{n}^{2}\right)=0, \quad n=1,2, \ldots, N .
$$


The resulting $N$ eigenfunctions $\phi_{j, n}=e^{-m_{n}^{2} / 2} L_{j-1}\left(m_{n}^{2}\right), j=1,2, \ldots, N$ with $n=$ $1,2, \ldots, N$ form a complete orthogonal set, but there are no longer properly normalized to 1. Using the Darboux-Christoffel formula for the Laguerre polynomials we find that the correctly normalized orthogonal complete set of eigenvectors is given by

$$
\phi_{j, n}=\frac{m_{n}}{N\left|L_{N-1}\left(m_{n}^{2}\right)\right|} L_{j-1}\left(m_{n}^{2}\right), \quad j=1,2, \ldots, N, \quad n=1,2, \ldots, N .
$$

Then the potential between charges located at the lattice-sites $j$ and $k$, and separated by a distance $r$ is

$$
V_{j, k}(r)=-\frac{g_{4}^{2}(N+1)}{r} \sum_{n=1}^{N} \frac{m_{n}^{2}}{N^{2} L_{N-1}^{2}\left(m_{n}^{2}\right)} L_{j-1}\left(m_{n}^{2}\right) L_{k-1}\left(m_{n}^{2}\right) e^{-m_{n} v r} .
$$

As an example consider the simplest non-trivial case with $N=2$. The two roots of $L_{2}\left(m^{2}\right)=0$ are given by $m_{\mp}^{2}=2 \mp \sqrt{2}$. Using the fact that $L_{0}(x)=1$ and $L_{1}(x)=1-x$ we find that the two eigenfunctions forming a complete orthonormal set according to (8), are

$$
\phi_{1, \mp}=\frac{m_{ \pm}}{2}, \quad \phi_{2, \mp}= \pm \frac{m_{\mp}}{2} .
$$

The potential between charges contains of course only Yukawa-type terms. We have

$$
V_{i j}(r)=-\frac{g_{4}^{2}}{r}\left(a_{i j}^{-} e^{-m_{-} v r}+a_{i j}^{+} e^{-m_{+} v r}\right), \quad i, j=1,2
$$

where the $2 \times 2$ matrices $\mathbf{a}^{\mp}$ are given by

$$
\mathbf{a}^{\mp}=\frac{3}{4}\left(\begin{array}{cc}
2 \pm \sqrt{2} & \pm \sqrt{2} \\
\pm \sqrt{2} & 2 \mp \sqrt{2}
\end{array}\right) \text {. }
$$

Keeping the zero mode: Let us now impose the boundary condition (6), that is $\phi_{N+2}=$ $\overline{\phi_{N+1}}$, or using the properties of the Laguerre polynomials, $\phi_{N+2}^{\prime}=0$. That determines $N$ nonzero mass eigenvalues $m_{n}^{2}, n=1,2, \ldots, N$ from the condition

$$
L_{N+1}^{\prime}\left(m_{n}^{2}\right)=0 \Rightarrow L_{N+1}\left(m_{n}^{2}\right)=L_{N}\left(m_{n}^{2}\right)=L_{N}^{\prime}\left(m_{n}^{2}\right), \quad n=1,2, \ldots, N .
$$

However, there is also the zero mode which is preserved by the boundary condition. As before, using the Darboux-Christoffel formula for the Laguerre polynomials we find that the correctly normalized orthogonal complete set of eigenvectors is given by

$$
\begin{aligned}
\phi_{j, n} & =\frac{1}{\sqrt{N+1}\left|L_{N}\left(m_{n}^{2}\right)\right|} L_{j-1}\left(m_{n}^{2}\right), \quad j=1,2, \ldots, N+1, \quad n=1,2, \ldots, N, \\
\phi_{j, 0} & =\frac{1}{\sqrt{N+1}}, \quad j=1,2, \ldots, N+1 .
\end{aligned}
$$

Then the potential between charges at sites $j$ and $k$ has a Coulombic as well as Yukawa terms

$$
V_{j, k}=-\frac{g_{4}^{2}}{r}-\frac{g_{4}^{2}}{r} \sum_{n=1}^{N} \frac{1}{L_{N}^{2}\left(m_{n}^{2}\right)} L_{j-1}\left(m_{n}^{2}\right) L_{k-1}\left(m_{n}^{2}\right) e^{-m_{n} v r} .
$$


As an example consider again the case of $N=2$. Now there are three mass eigenvalues given by $m_{0}^{2}=0$ and the two zeroes of the algebraic equation $L_{3}^{\prime}\left(m^{2}\right)=0$ which are $m_{\mp}^{2}=3 \mp \sqrt{3}$. The corresponding eigenfunctions are

$$
\phi_{1, \mp}=\frac{\sqrt{3} \pm 1}{2 \sqrt{3}}, \quad \phi_{2, \mp}=\frac{ \pm 1-\sqrt{3}}{2 \sqrt{3}}, \quad \phi_{3, \mp}=\mp \frac{1}{\sqrt{3}}, \quad \phi_{j, 0}=\frac{1}{\sqrt{3}} .
$$

The potential between charges is

$$
V_{j, k}(r)=-\frac{g_{4}^{2}}{r}-\frac{g_{4}^{2}}{r}\left(b_{j k}^{-} e^{-m_{-} v r}+b_{j k}^{+} e^{-m_{+} v r}\right), \quad j, k=1,2,3,
$$

where the $3 \times 3$ matrices $\mathbf{b}^{\mp}$ are given by

$$
\mathbf{b}^{\mp}=\frac{1}{2}\left(\begin{array}{ccc}
2 \pm \sqrt{3} & -1 & -1 \mp \sqrt{3} \\
-1 & 2 \mp \sqrt{3} & \pm \sqrt{3}-1 \\
-1 \mp \sqrt{3} & \pm \sqrt{3}-1 & 2
\end{array}\right)
$$

Finally, we note that when $N$ becomes very large, the spectrum in both cases that we have considered becomes continuous and the above expressions approach the corresponding ones in (39) and (40). This can be seen using the property of the Laguerre polynomials

$$
L_{n}(x)=\frac{e^{x / 2}}{\sqrt{\pi}(n x)^{1 / 4}} \cos (2 \sqrt{n x}-\pi / 4)+\mathcal{O}\left(n^{-3 / 4}\right), \quad \text { for } \quad n \gg 1 .
$$

\subsection{Example within the continuous approximation}

We consider now cases where the lattice is already treated in the continuum approximation where a description in terms of a gauge theory in a five-dimensional warped spacetime (16) is valid, namely when $1 \ll v r \ll N$. The distribution of vev's might be such that the mass parameter that sets the scale at these distances is not $v$ itself, but some other mass parameter $M_{0}$. This is assumed to be such that $v / N \ll M_{0} \ll v$ which implies that the corresponding length scale $1 / M_{0}$ is within the allowed distances for the continuum approximation to be valid.

First, let us consider the case with $f\left(x_{5}\right)=2 M_{0} x_{5}, x_{5} \geq 0$. Applying the general formalism we easily find that the variable $x_{5}$ and the variable $z$ that enters in (18) are related by $2 M_{0} x_{5}=e^{2 M_{0} z}$, with $-\infty<z<\infty$ and that the function $A(z)=2 M_{0} z$. Therefore the Schrödinger potential in (20) is just a constant, i.e. $V_{\text {Sch. }}=M_{0}^{2}$. That defines a mass gap since in order to have normalizable wavefunctions we must require that $M^{2} \geq M_{0}^{2}$. We obtain

$$
\phi_{M}(z)=\frac{e^{-M_{0} z}}{2 \sqrt{\pi}}\left(M^{2}-M_{0}^{2}\right)^{-1 / 4} e^{i\left(M^{2}-M_{0}^{2}\right)^{1 / 2} z} .
$$

Hence, the potential between charges at positions $z$ and $z^{\prime}$ in the fifth dimension is

$$
V_{z, z^{\prime}}=-\frac{g_{5}^{2} M_{0} e^{-M_{0}\left(z+z^{\prime}\right)}}{\pi R} K_{1}\left(M_{0} R\right), \quad R=\sqrt{r^{2}+\left(z-z^{\prime}\right)^{2}},
$$


where $K_{1}$ is the modified Bessel function of order one. If we consider distances much smaller than the characteristic scale of the mass gap $1 / M_{0}$, then we obtain indeed the behaviour (21). Instead, in the opposite limit of distances much larger than $1 / M_{0}$ we find that

$$
V_{z, z^{\prime}}(r) \simeq-\frac{g_{5}^{2}}{\sqrt{2 \pi}} e^{-M_{0}\left(z+z^{\prime}\right)} \frac{M_{0}^{2}}{\left(M_{0} R\right)^{3 / 2}} e^{-M_{0} R}, \quad \text { for } \quad 1 / M_{0} \ll R \ll N / v .
$$

Naturally, the range of Yukawa interaction is set by the mass gap, whereas the strength has the $1 / r^{3 / 2}$ behaviour as noted in footnote 2 .

Also, the value of the mass gap $M_{0}$ is in agreement with what was noted in footnote 5 for the exact lattice treatment of the vev distribution $v_{j}=v j$, after we set $M_{0}=v / 2$.

\subsection{Another example within the continuous approximation}

A generalization of the previous case which however does not lead to a mass gap for the spectrum, is to take $f\left(x_{5}\right)=\left(2(\nu+1) M_{0} x_{5}\right)^{\frac{2 \nu+1}{2(\nu+1)}}$ with $x_{5} \geq 0$ and where the exponent of $x_{5}$ is introduced as such for later notational convenience. We find that the variables $x_{5}$ and $z$ are related as $2(\nu+1) M_{0} x_{5}=\left(M_{0} z\right)^{2(\nu+1)}$ and also that $e^{A}=\left(M_{0} z\right)^{2 \nu+1}$. Hence, the Scrhödinger potential takes the form

$$
V_{\text {Sch. }}=\frac{\nu^{2}-1 / 4}{z^{2}}
$$

from which we conclude that the mass spectrum is continuous without a mass gap. The eigenfunctions are given in terms of Bessel functions as

$$
\phi_{M}(z)=\frac{1}{\sqrt{2 M_{0}}}\left(M_{0} z\right)^{-\nu} J_{\nu}(M z) .
$$

It turns out that the potential between charges at the same position $z$ in the fifth dimension is computed in terms of a hypergeometric function as (for simplicity we do not deal with the case of charges at different positions in the fifth dimension)

$$
\begin{gathered}
V_{z, z}(r)=-\frac{2 \Gamma(\nu+3 / 2)}{\sqrt{\pi} \Gamma(\nu+1)} \frac{g_{5}^{2} / M_{0}^{2 \nu+1}}{r^{2}\left(r^{2}+4 z^{2}\right)^{\nu+1 / 2}} F\left(\nu+1 / 2, \nu-1 / 2,2 \nu+1, \frac{4 z^{2}}{r^{2}+4 z^{2}}\right), \\
\text { for } 1 / v \ll r \ll N / v .
\end{gathered}
$$

We also note the limiting case

$$
V_{z, z}(r)=-\frac{2 \Gamma(\nu+3 / 2)}{\sqrt{\pi} \Gamma(\nu+1)} \frac{g_{5}^{2} / M_{0}^{2 \nu+1}}{r^{2 \nu+3}}, \quad \text { for } \quad 1 / v \ll z \ll r
$$

and that for $r \ll z$ we confirm the behaviour (21).

\footnotetext{
${ }^{6}$ Note that, for $M_{0}=v / 2$ and $\nu=0$ we have that $v_{j}=v \sqrt{j}$. Then indeed the wavefunction (60) agrees with (42).
} 


\section{Strong coupling considerations}

We would like to study similar effects at strong coupling using the AdS/CFT correspondence [18]. We will see that our findings and the emergence of an effective fifth dimension are in precise agreement with the gauge theory considerations so far.

The clearest discussion for our purposes can be done for the Coulomb branch of the $\mathcal{N}=4 \mathrm{SYM}$ theory with gauge group $S U(N k)$. This theory has six scalars $X^{I}$, $I=1,2, \ldots, 6$ in the adjoint of the gauge group. We will reanalyze and refine the model introduced in [2] and we will study the potential between a quark and an antiquark. We give vev's to two of the six scalars in the theory in a way that the original $S O(6)$ global invariance of the theory that rotates the six scalars, breaks to $S O(4) \times Z_{N}$. We may represent these scalars as $N k \times N k$ traceless matrices. In general, since the scalar potential in the SYM action is proportional to $\sum_{I<J} \operatorname{tr}\left[X^{I}, X^{J}\right]^{2}$ we can choose the vev's along the diagonal so that the potential is zero. That preserves sixteen supercharges and breaks the conformal invariance. We arrange the $N k \times N k$ matrices $X^{I}$ 's in the form of block-diagonal matrices with $N$ diagonal $k \times k$ blocks, where in each block the vev's are the same. The $\mathcal{N}=4$ SYM theory action contains the term $\sum_{I=1}^{6} \operatorname{tr}\left(D_{\mu} \Phi^{I}\right)^{2}$ which couples the gauge fields and the six scalars. It is convenient to choose the standard real basis for the $S U(N k)$ generators $J_{i j}=e_{i j}-\frac{1}{N k} \delta_{i j} I_{N k \times N k}$, where the matrix elements of the matrices $e_{i j}$ are $\left(e_{i j}\right)_{m n}=\delta_{i m} \delta_{j n}$. Hence, we give vev's to the scalars represented

by the six-dimensional vector $\vec{\Phi}$, as $\vec{\Phi}=h_{i} \vec{X}_{\text {vev }}^{i}$, where $h_{i}=J_{i i}$ are the diagonal Cartan generators. The masses of the gauge fields arise from the term $\mathcal{L}_{\text {mass }} \sim \sum_{I=1}^{6} \operatorname{tr}\left[\Phi^{I}, A_{\mu}\right]^{2}$. After we define $A_{\mu}=A_{\mu}^{i j} J_{i j}$ we have

$$
\mathcal{L}_{\text {mass }} \sim k \sum_{i, j=0}^{N-1}\left(\vec{X}_{\mathrm{vev}}^{i} \cdot \vec{X}_{\mathrm{vev}}^{j}-\vec{X}_{\mathrm{vev}}^{i} \cdot \vec{X}_{\mathrm{vev}}^{i}\right) A_{\mu}^{i j} A^{\mu, j i} .
$$

This basic formula can now be applied to specific cases. As in [2] we choose for the vev's in the $j$ th block the values

$$
\vec{X}_{\mathrm{vev}}^{j}=\left(0,0,0,0, r_{0} \cos (2 \pi j / N), r_{0} \sin (2 \pi j / N)\right), \quad j=0,1, \ldots, N-1
$$

These form an $N$-sided polygon enclosed by a circle in the $x_{5}-x_{6}$ plane of radius $r_{0}$. Then we find that (63) becomes

$$
\mathcal{L}_{\text {mass }} \sim r_{0}^{2} k \sum_{i, j=0}^{N-1} \sin ^{2}(\pi(j-i) / N) A_{\mu}^{i j} A^{\mu, j i} .
$$

From this we read off the masses as

$$
M_{n} \sim r_{0} \sqrt{k} \sin (\pi n / N), \quad n=0,1, \ldots, N-1
$$

which are the same as the ones we found in (23). They have a degeneracy $d_{n}$ which is: $d_{0}=N-1$ and $d_{n}=2(N-n)$, for $n \geq 1$ (Note that $\sum_{n=0}^{N-1} d_{n}=N^{2}-1$ ). Using the 
fact that $M_{n}=M_{N-n}$ it is easily seen that the expression for the potential is given by $V=-g_{4}^{2} /(N r) \sum_{n=0}^{N-1} d_{n} e^{-M_{n} r}=-g_{4}^{2} / r\left(\sum_{n=0}^{N-1} e^{-M_{n} r}-1 / N\right)$, which, for large enough $N$, is the same as the potential (10) corresponding to the mass formulae in (23) (for test particles at the same lattice cite). That makes apparent that the model in [2] and the construction of [4, 5] (reviewed in subsection 3.1) describe essentially the same Physics.

The advantage of having a D3-brane realization in a model with an emerging latticed fifth dimension, is that we may consider the theory at strong coupling using the AdS/CFT correspondence. In the type-IIB theory we consider a large number $N k$ of D3-branes that are distributed in the transverse to the branes six-dimensional space according to (64). Namely, the gravitational metric that we will consider is

$$
d s^{2}=H^{-1 / 2} \eta_{\mu \nu} d y^{\mu} d y^{\nu}+H^{1 / 2}\left(d x_{1}^{2}+\cdots+d x_{6}^{2}\right),
$$

where $y^{\mu}, \mu=0,1,2,3$ are the parallel to the brane directions. The harmonic function $H$ in the six-dimensional space spanned by $x_{i}, i=1,2, \ldots, 6$ is given by

$$
H=\sum_{j=0}^{N-1} \frac{R_{4}^{4}}{\left|\vec{x}-\vec{X}_{\mathrm{vev}}^{j}\right|^{4}}
$$

where $R_{4}^{4}=4 \pi g_{4}^{2} k$ is the 't Hooft coupling associated with the $S U(k)$ gauge theory. We see that the vev's in the field theory (64) become the centers in the harmonic function above. We emphasize that the supergravity approximation is valid even close to each one of the centers if we choose that $R_{4}^{4} \gg 1$ and also small values for $g_{4}^{2}$ which implies that there are many branes located at each center, i.e. $k \gg 1$. The number of such centers $N$ need not be large.

The harmonic function $H$ has been computed in [2]. It takes the form

$$
H=R^{4} \frac{U^{2}+r_{0}^{2}}{\left(\left(U^{2}+r_{0}^{2}\right)^{2}-4 r_{0}^{2} u^{2}\right)^{3 / 2}} \Sigma_{N},
$$

where $R^{4}=4 \pi g_{4}^{2} k N$. Also $U^{2}=x_{1}^{2}+\ldots+x_{6}^{2}, u^{2}=x_{5}^{2}+x_{6}^{2}$ and

$$
\Sigma_{N}=\frac{\sinh N x}{\cosh N x-\cos N \psi}+N \frac{\left(\left(U^{2}+r_{0}^{2}\right)^{2}-4 r_{0}^{2} u^{2}\right)^{1 / 2}}{U^{2}+r_{0}^{2}} \frac{\cosh N x \cos N \psi-1}{(\cosh N x-\cos N \psi)^{2}},
$$

where $\psi$ is the polar angle in the $x_{5}-x_{6}$ plane and

$$
e^{x}=\frac{U^{2}+r_{0}^{2}}{2 r_{0} u}+\sqrt{\left(\frac{U^{2}+r_{0}^{2}}{2 r_{0} u}\right)^{2}-1}
$$

Notice that $H$ explicitly processes the symmetry $S O(4)$ in the four-dimensional subspace spanned by $x_{1}, x_{2}, x_{3}$ and $x_{4}$ as well as the $Z_{N}$ symmetry under the shift $\psi \rightarrow \psi+2 \pi / N$.

The potential between a pair of heavy quark and antiquark separated by a distance $L$ is found by minimizing the Nambu-Goto action of a fundamental string in the supergravity background (67). We will not give details as the necessary techniques we

\footnotetext{
${ }^{7}$ We denote the distance between the charges by $L$ in order to confirm with standard notation in the AdS/CFT literature. This is nothing but the distance denoted by $r$ in the previous part of the paper.
} 
first developed for the conformal case in [19]. For backgrounds of the type that we have here we will use eqs. (5)-(7) in [3]. The trajectory that minimizes the action lies on the $x_{5}-x_{6}$ plane where $u=U$. However, not every angle $\psi$ is allowed, but only those with $\sin (N \psi)=0$. All angles satisfying this condition are equivalent because of the $Z_{N}$ symmetry. For concreteness we may choose the trajectory along the $x_{5}$-axis (for $\psi=0$ and $u=U)$. Let's define the function $f=R^{4} / H$. Then for the trajectory that we are interested in we have

$$
\begin{aligned}
f(U) & =\frac{\left(U^{2}-r_{0}^{2}\right)^{3}}{U^{2}+r_{0}^{2}} \frac{1}{\Sigma} \\
\Sigma & =\frac{\left(U / r_{0}\right)^{N}+1}{\left(U / r_{0}\right)^{N}-1}+\frac{2 N}{\left(U / r_{0}\right)^{N}+\left(r_{0} / U\right)^{N}-2} \frac{\left(U / r_{0}\right)^{2}-1}{\left(U / r_{0}\right)^{2}+1}
\end{aligned}
$$

where we have used the fact that $e^{x}=U / r_{0}$, when $u=U$ and $\psi=0$. Then the integrals for the length $L$ and the energy $V$ are given by

$$
L=2 R^{2} \int_{U_{0}}^{\infty} d U \sqrt{\frac{f\left(U_{0}\right)}{f(U)\left(f(U)-f\left(U_{0}\right)\right)}}
$$

and

$$
V=\frac{1}{\pi} \int_{U_{0}}^{\infty} d U\left[\sqrt{\frac{f(U)}{f(U)-f\left(U_{0}\right)}}-1\right]-\frac{1}{\pi}\left(U-r_{0}\right)
$$

where $U_{0}$ denotes the lowest value of $U$ that can be reached by the string geodesic. In principle, we have to evaluate the integrals and try to express $V$ as a function of $L$. This is impossible in general though it is easy to deduce that $V$ is a monotonously increasing functions of $L$ from $-\infty$ to 0 in the interval $L \in(0, \infty)$.

\subsection{Examining various energy regimes}

As usual, in the AdS/CFT correspondence large (small) distances in the five-dimensional geometry, corresponds to small (large) distances in the gauge theory side [20]. The variable $U$ represents a distance in the AdS side and it is interpreted as an energy variable in the gauge theory side. It is instructive to find the various limits of the metric (67) and of the quark-antiquark potential, by examining the behaviour of the harmonic function $H$ as we go from the UV to the IR. The UV corresponds to energies large enough so that the vev's can be neglected. The deep IR corresponds to energies very close to the vev's.

\subsubsection{Behaviour for $U \gg r_{0}$}

In the UV for $U \gg r_{0}$ since the harmonic takes the form

$$
H \simeq \frac{R^{4}}{U^{4}}, \quad \text { for } \quad U \gg r_{0}
$$


the metric becomes that for $A d S_{5} \times S^{5}$ with each factor having radius $R$ in string units, Then the original $S O(6)$ global symmetry of the metric is restored. That corresponds to the fact that in the deep UV the values of the vev's can be neglected and we should be describing an $S U(N k)$ SYM theory. In this regime the integrals can be evaluated and give for the potential energy between a quark and antiquark the results of [19. Hence we have

$$
V \simeq-2\left(\frac{\Gamma(3 / 4)}{\Gamma(1 / 4)}\right)^{2} \frac{R_{4}^{2} \sqrt{N}}{L}, \quad L \ll \frac{R_{4}^{2} \sqrt{N}}{r_{0}} .
$$

This behaviour is similar to the one found from pure gauge theory considerations in (15) for the interaction of charges at the same site $j=k$.

\subsubsection{Behaviour for $U-r_{0} \gg r_{0} / N$}

For distances from the location of the centers much larger than the distance between the different centers we may approximate the harmonic function by [2]

$$
H=R^{4} \frac{U^{2}+r_{0}^{2}}{\left(\left(U^{2}+r_{0}^{2}\right)^{2}-4 r_{0}^{2} u^{2}\right)^{3 / 2}}, \quad \text { for } U-r_{0} \gg r_{0} / N,
$$

since then $\Sigma_{N} \simeq 1$. Then we see that the discrete part $Z_{N}$ of the symmetry group $S O(4) \times Z_{N}$ becomes a continuous $U(1)$. Then the resulting integrals for the length and the energy are quite complicated (see eqs. (A.6) and (A.7) of [3]) and it does not seem possible to compute them explicitly, let alone to explicitly obtain the energy as a function of the separation length. This approximation is valid for $L \ll R_{4}^{2} N / r_{0}$. The situation here is similar to the one described from a gauge theory point of view by eq. (24) (for $j=k)$.

As a separate remark we note that the metric (67) with the above harmonic (77) has a ring singularity defined by the equations $U^{2}=u^{2}=x_{5}^{2}+x_{6}^{2}=r_{0}^{2}$. It is obvious that this is an artifact of the continuous approximation which is not valid near the singularity, where the harmonic which should be used is given by (80) below and the metric then is completely non-singular. The same resolution of singularities applies to solutions that have been used for studies of the Coulomb branch of the $\mathcal{N}=4$ SYM gauge theory in, for instance, [3, 21, 22. These solutions, take the form (67), but unlike (69), the distribution of D3-branes is continuous instead of discrete. It is obvious that the solutions will become non-singular if we replace the continuous by a discrete distribution of D3-branes with the given continuous limit. An example of that was already given in the appendix of [2] representing the discrete version of a continuous distribution of D3-branes on a disc.

As another side remark we note that the solution with harmonic (69) as well as its continuous approximation (77), do not have a five-dimensional gauged supergravity origin. 


\subsubsection{Behaviour for $r_{0} / N \ll U-r_{0} \ll r_{0}$}

For distances from the centers much larger than the distance between the different centers, but also much smaller than the radius of the circle, we may approximated the harmonic function by

$$
H \simeq \frac{R^{4}}{4 r_{0} U^{3}}, \quad \text { for } \quad r_{0} / N \ll U-r_{0} \ll r_{0} .
$$

In this case we have a smeared D3-brane solution along one transverse direction which is T-dual to a D4-brane solution. Also the global symmetry has been enhanced from an $S O(4) \times U(1)$ into an $S O(5)$. Since a D4-brane solution corresponds to a five-dimensional theory we expect that there will be a $1 / L^{2}$ behaviour for the quark-antiquark potential. Indeed we obtain the result [3]

$$
V \simeq-4 \sqrt{\pi}\left(\frac{\Gamma(2 / 3)}{\Gamma(1 / 6)}\right)^{3} \frac{R_{4}^{4} N}{r_{0} L^{2}}, \quad \text { for } \quad \frac{R_{4}^{2} \sqrt{N}}{r_{0}} \ll L \ll \frac{R_{4}^{2} N}{r_{0}} .
$$

The behaviour is similar to (25) from the usual gauge theory point of view.

\subsubsection{Behaviour for $U-r_{0} \ll r_{0} / N$}

In the deep IR for energy scales very close to the vev's

$$
H \simeq \frac{R_{4}^{2}}{U^{4}}, \quad \text { for } \quad U-r_{0} \ll r_{0} / N,
$$

where $R_{4}^{4}=4 \pi g_{4} k$ is the 't Hooft coupling for an $S U(k)$ gauge theory. Then we have

$$
V \simeq-2\left(\frac{\Gamma(3 / 4)}{\Gamma(1 / 4)}\right)^{2} \frac{R_{4}^{2}}{L}, \quad \text { for } \quad L \gg \frac{R_{4}^{2} N}{r_{0}} .
$$

This is a Coulombic behaviour for a gauge group $S U(k)$ and is similar to that in (13) from the usual gauge theory point of view.

\subsubsection{Discrete infinite array}

Finally, we may also find a regime where the original circular discrete array is approximated by a discrete infinite array on a straight line. This is possible for distances from the centers much smaller that the radius of the circle. From the gauge theory point of view at weak coupling the analogous potential is described by (26). The solution has a metric given by (67) with harmonic

$$
\begin{aligned}
H & =\sum_{j=-\infty}^{\infty} \frac{R_{4}^{4}}{\left(U^{2}+\left(x_{6}-2 \pi v j\right)^{2}\right)^{2}} \\
& =\frac{R_{4}^{4}}{4 U^{3} v^{2}}\left(\frac{v \sinh (U / v)}{\cosh (U / v)-\cos \left(x_{6} / v\right)}+U \frac{\cosh (U / v) \cos \left(x_{6} / v\right)-1}{\left(\cosh (U / v)-\cos \left(x_{6} / v\right)\right)^{2}}\right)
\end{aligned}
$$


where $U^{2}=x_{1}^{2}+x_{2}^{2}+\ldots+x_{5}^{2}$ and $v=r_{0} / N$. Translated into separation distances for the quark-antiquark pair, this approximation is valid when $L \gg R_{4}^{2} \sqrt{N} / r_{0}$. The trajectory that we will use to compute the potential amounts to setting $x_{6}=0$. Then we utilize the general formulae (73) and (74) with $R$ replaced by $R_{4}$ and the function $f$ being given by

$$
f(U)=4 v U^{3}\left(\operatorname{coth}(U /(2 v))+\frac{U /(2 v)}{\sinh ^{2}(U /(2 v))}\right)^{-1} .
$$

As we move from $U \gg v$ to $U \ll v$ this case interpolates between those described before by (79) and (81). It is not possible to compute the energy as a function of the separation explicitly, but we may use the above expression in order to find the first correction to the $1 / L^{2}$ behaviour in (79). Expanding (83) for $U \gg v$ and keeping the two leading terms we find that

$$
V=-4 \sqrt{\pi}\left(\frac{\Gamma(2 / 3)}{\Gamma(1 / 6)}\right)^{3} \frac{R_{4}^{4}}{v L^{2}}\left(1+c_{1} \frac{R_{4}^{6}}{v^{3} L^{3}} e^{-c_{2} \frac{R_{4}^{4}}{v^{2} L^{2}}}+\cdots\right)
$$

where the $c_{1}$ and $c_{2}$ are two constants of oder 1 . Since $1 \ll \frac{R_{4}^{4}}{v^{2} L^{2}} \ll N$ the corrections to the leading $1 / L^{2}$ behaviour are small. However, they do not obey a power-like law as the corrections to (25) (noted after (10)). Similarly, the corrections to (81) are found by expanding (83) for $U \ll v$. It is easy to see that these corrections are organized in powers of $\frac{R_{4}^{2} N}{r_{0} L} \ll 1$, in contrast to the corrections to (13) which are of the Yukawa type (noted again below (10)). Hence, it seems that the mechanisms for developing an effective fifth dimension are different at weak and strong coupling.

In will be interesting to further extend the results of this paper in order to incorporate fermions and to investigate the renormalization of the gauge coupling constant. Also an extension to non-commutative gauge theories could be of some interest.

\section{Acknowledgements}

I would like to thank A. Kehagias for a discussion. This research was supported by the European Union under contracts TMR-ERBFMRX-CT96-0045 and -0090, by the Swiss Office for Education and Science, by the Swiss National Foundation and by the contract HPRN-CT-2000-00122.

\section{A Varying gauge coupling}

In this appendix we first study differential operators defined on the lattice of the type

$$
T_{j}=q_{j}^{\dagger} q_{j}, \quad j=0,1, \ldots, N,
$$

for some operator $q_{j}$ and which have energy eigenvalues that are positive semi-definite, i.e. $M^{2} \geq 0$. In the case of the operator (雨) discussed in the main text we have that

${ }^{8}$ Precisely, $c_{1}=\sqrt{8 \pi^{3}}\left(\frac{8}{3 \sqrt{6}}-1\right)\left(\frac{\Gamma(2 / 3)}{\Gamma(1 / 6)}\right)^{2} \simeq 0.083$ and $c_{2}=4 \pi\left(\frac{\Gamma(2 / 3)}{\Gamma(1 / 6)}\right)^{2} \simeq 0.744$. 
$q_{j}=v f_{j}\left(e^{d_{j}}-1\right)$, as already noted. If a state $\phi_{j, 0}$ satisfies the equation $q_{j} \phi_{j, 0}=0$, then automatically we have a solution of $T_{j} \phi_{j, 0}=0$ corresponding to a zero mass eigenvalue. Define now the lattice differential operator $\tilde{T}_{j}$ obtained by reversing the order of $q_{j}$ and $q_{j}^{\dagger}$ in $(\mathrm{A} .1)$

$$
\tilde{T}_{j}=q_{j} q_{j}^{\dagger}, \quad j=0,1, \ldots, N .
$$

The discussion next is completely parallel to that in supersymmetric quantum mechanics (SQM) [17]. The spectra and eigenfunctions of $M_{n}^{2}$ and $\tilde{M}_{n}^{2}$ of $T_{j}$ and $\tilde{T}_{j}$

$$
\begin{aligned}
& \tilde{M}_{n}^{2}=M_{n+1}^{2}, \quad M_{0}^{2}=0, \\
& \tilde{\phi}_{j, n}=\frac{1}{M_{n+1}} q_{j} \phi_{j, n+1}, \\
& \phi_{j, n+1}=\frac{1}{\tilde{M}_{n}} q_{j}^{\dagger} \tilde{\phi}_{j, n} .
\end{aligned}
$$

Hence, the spectra of the operators $T_{j}$ and $\tilde{T}_{j}$ are identical except for the zero mode. As we have seen, boundary conditions may project it out of the spectrum in which case the spectra are identical. Moreover, acting with $q_{j}\left(q_{j}^{\dagger}\right)$ we may convert an eigenvector of $T_{j}$ $\left(\tilde{T}_{j}\right)$ into an eigenvector of $\tilde{T}_{j}\left(T_{j}\right)$.

Let us define the matrices

$$
H_{j}=\left(\begin{array}{cc}
T_{j} & 0 \\
0 & \tilde{T}_{j}
\end{array}\right), \quad Q_{j}=\left(\begin{array}{cc}
0 & 0 \\
q_{j} & 0
\end{array}\right), \quad Q_{j}^{\dagger}=\left(\begin{array}{cc}
0 & q_{j}^{\dagger} \\
0 & 0
\end{array}\right) .
$$

It is trivial that these obey the closed superalgbebra

$$
\left[H_{j}, Q_{j}\right]=\left[H_{j}, Q_{j}^{\dagger}\right]=\left\{Q_{j}, Q_{j}\right\}=\left\{Q_{j}^{\dagger}, Q_{j}^{\dagger}\right\}=0, \quad\left\{Q_{j}, Q_{j}^{\dagger}\right\}=H_{j}
$$

In terms of this superalgebra, the fact that $Q_{j}$ and $Q_{j}^{\dagger}$ commute with $H_{j}$ is responsible for the degeneracy of the spectra.

As an application of the above general results, let us consider again the low energy effective action in the lattice (1), but now with varying gauge coupling $g_{4, j}^{2}$ and Higgs values $v_{j}$. The relevant term derived from (1) corresponding to the gauge field fluctuations is

$$
S=-\frac{1}{4(N+1)} \int d^{4} x \sum_{j=0}^{N} \operatorname{tr}\left(F_{\mu \nu}^{j}\right)^{2}+\sum_{j=0}^{N-1} v_{j}^{2} \operatorname{tr}\left(g_{4, j+1} A_{\mu}^{j+1}-g_{4, j} A_{\mu}^{j}\right)^{2} .
$$

Consider first the case of constant vev's $v_{j}=v$. The mass term above can be written as

$$
\sum_{j=0}^{N} A_{\mu}^{j} \tilde{T}_{j} A_{\mu}^{j}
$$

where the operator $\tilde{T}_{j}$ is defined as

$$
\tilde{T}_{j}=v^{2} g_{4, j}\left(e^{-d_{j}}-1\right)\left(e^{d_{j}}-1\right) g_{4, j}=-4 v^{2} g_{4, j} \sinh ^{2}\left(d_{j} / 2\right) g_{4, j} .
$$


Hence, the operator $\tilde{T}_{j}$ has precisely the form (A.2) with $q_{j}=g_{4, j}\left(e^{-d_{j}}-1\right)$. Using the formalism that we developed we may map this problem into a problem for the superpartner operator $T_{j}$ in (A.1). Comparing with the case of constant gauge coupling and varying vev's considered in section 2 , we find that they are identical after we identify $v^{2} g_{4, j+\frac{1}{2}}^{2}$ by $v^{2} f_{j-\frac{1}{2}}^{2}$.

As a specific application consider the case of couplings $g_{4, j}=g_{4} \sqrt{j}, j \geq 1$. After using the results of subsection 3.3 we find that the set of eigenfunctions of the operator $T_{j}$ are

$$
\phi_{j} \sim L_{j}\left(m^{2}\right), \quad m=\frac{M}{g_{4} v} .
$$

Using (A.3) we find that the eigenfunctions of the operator (A.8) are

$$
\tilde{\phi}_{j} \sim \sqrt{j}\left(L_{j-1}\left(m^{2}\right)-L_{j}\left(m^{2}\right)\right)
$$

We may easily verify, using recursion relations for the Laguerre polynomials, that this solution satisfies the eigenvalue equation for the operator $\tilde{T}_{j}$ in (A.8). We have left undetermined the overall normalization constant, since this would depend on the boundary conditions that we will impose. Note that a choice of a boundary condition for either $\phi_{j}$ or $\tilde{\phi}_{j}$ implies the boundary condition for the other, if we want that the mass spectra are also related according to (A.3). Of course we may still impose independent boundary conditions in which case the two spectra are not related.

Finally, we note that in the most general case of varying vev's and gauge couplings, the operator $T_{j}$ still takes the form (A.1) with $q_{j}=v_{j}\left(e^{d_{j}}-1\right) g_{4, j}$.

\section{References}

[1] I. Antoniadis, S. Dimopoulos and G. Dvali, Nucl. Phys. B516 (1998) 70, hep-ph/9710204; N. Arkani-Hamed, S. Dimopoulos and G. Dvali, Phys. Lett. B429 (1998) 263, hep-ph/9803315; For related experiments, see for instance,J.C. Long, H.W. Chan and J.C. Price, Nucl. Phys. B539 (1999) 23, hep-ph/9805217.

[2] K. Sfetsos, JHEP 01 (1999) 015, hep-th/9811167.

[3] A. Brandhuber and K. Sfetsos, Adv. Theor. Math. Phys. 3 (1999) 851, hep-th/9906201.

[4] N. Arkani-Hamed, A. G. Cohen and H. Georgi, (De)constructing dimensions, Phys. Rev. Lett. 86 (2001) 4757 hep-th/0104005.

[5] C.T. Hill, S. Pokorski and J. Wang, Gauge invariant effective Lagrangian for KaluzaKlein modes, hep-th/0104035.

[6] M.B. Halpern and W. Siegel, Phys. Rev. D11 (1975) 2967. 
[7] Y.K. Fu and H.B. Nielsen, Nucl. Phys. B236 (1984) 167.

[8] Y.K. Fu and H. B. Nielsen, Nucl. Phys. B254 (1985) 127.

[9] A. Hulsebos, C.P. Korthals-Altes and S. Nicolis, Nucl. Phys. B450 (1995) 437, hep-th/9406003; P. Dimopoulos, K. Farakos, C.P. Korthals-Altes, G. Koutsoumbas and S. Nicolis, JHEP 0102 (2001) 005, hep-lat/0012028.

[10] H. Cheng, C.T. Hill, S. Pokorski and J. Wang, The standard model in the latticized bulk, hep-th/0104179.

[11] N. Arkani-Hamed, A. G. Cohen and H. Georgi, Electroweak symmetry breaking from dimensional deconstruction, hep-ph/0105239.

[12] H. Cheng, C. T.Hill and J. Wang, Dynamical electroweak breaking and latticized extra dimensions, hep-ph/0105323.

[13] C. Csaki, J. Erlich, C. Grojean and G. Kribs, 4D Constructions of Supersymmetric Extra Dimensions and Gaugino Mediation, hep-ph/0106044.

[14] H. D. Kim, To be (finite) or not to be, that is the question "Kaluza-Klein contribution to the Higgs mass, hep-ph/0106072.

[15] M. Alishahiha, (De)constructing dimensions and non-commutative geometry, hep-th/0105153.

[16] J. Dai and X. Song, Spontaneous symmetry broken condition in (de)constructing dimensions from noncommutative geometry, hep-ph/0105280.

[17] E. Witten, Nucl. Phys. B188 (1981) 513; For a review see, F. Cooper, A. Khare and U. Sukhatme, Phys. Rept. 251 (1995) 26, hep-th/9405029.

[18] J. Maldacena, Adv. Theor. Math. Phys. 2 (1998) 231, hep-th/9711200.

[19] J. Maldacena, Phys. Rev. Lett. 80 (1998) 4859 hep-th/9803002; S. Rey and J. Yee, Macroscopic strings as heavy quarks in large $N$ gauge theory and anti-de Sitter supergravity, hep-th/9803001.

[20] L. Susskind and E. Witten, The holographic bound in anti-de Sitter space, hep-th/9805114.

[21] I. Bakas and K. Sfetsos, Nucl. Phys. B573 (2000) 768, hep-th/9909041.

[22] D. Z. Freedman, S. S. Gubser, K. Pilch and N. P. Warner, JHEP 0007 (2000) 038, hep-th/9906194. 